\title{
Special announcement: Guidelines to the practice of anesthesia-Revised edition 2010
}

\author{
Richard N. Merchant, MD
}

Published online: 16 December 2009

(C) Canadian Anesthesiologists' Society 2009

The first Minimum Guidelines for the Standards of Practice of Anaesthesia (the Guidelines) was approved by the Council of the Canadian Anaesthetists' Society (the Society) on January 17, 1975 and published in booklet form in 1977. Several concurrent events drove the formulation of the Guidelines. ${ }^{1}$ The development of standards on "Anesthesia Services" by the Canadian Council on Hospital Accreditation (now Accreditation Canada) was an initial cause of concern in anesthesia circles, since it was perceived as an encroachment on "areas of responsibility". At about the same time, there were two unfortunate deaths under anesthesia that prompted the Canadian Medical Protective Association to consult with the Society and to advise them to propose minimum standards of anesthesia practice.

The Committee on Standards of Practice was directed to prepare such guidelines, and a final draft, much like the form we see today, was accepted by 1974 and published in 1975 as a policy of the Canadian Anaesthetists' Society. Dr. J. Earl Wynands, the President of the Society at that time, stated that the committee had produced "a major and definitive report that will have an impact on the practice and delivery of anaesthetic care in this country". All this, incidentally, occurred several years before the Harvard Standards were proposed in $1986,{ }^{2}$ so the Society can be regarded as an international leader in the field of standards development and patient safety.

The Guidelines were received with enthusiasm, although not universal support at that time, since some expressed

\section{R. N. Merchant, MD (ه)}

Department of Anesthesia and Perioperative Medicine, Royal Columbian Hospital, 330 Columbia St. E., New Westminster, BC V3L 3W7, Canada

e-mail: richard.merchant@ubc.ca concern that the Society had "set a standard that now had to be followed." However, a consensus evolved recognizing that the Guidelines were intended to represent the generally accepted standard of care and that they served as a "stimulus for improving the quality of anesthesia practice". The Guidelines were written in such a manner as to provide flexibility for modification as anesthesia practice evolves, as monitoring techniques become more sophisticated, and as new technologies become available. The Guidelines were modified periodically at irregular intervals until 1989 and annually since then. In an early revision of the Guidelines, the term "standards" was removed from the title of the document, emphasizing instead the term "guideline" to indicate that the document was intended to represent a "statement of principle rather than a standard or required level of conduct".

A contentious issue for some time was the role of general practitioners in providing anesthesia care in addition to Royal College trained specialists. This concern came about in the context of the general recognition that a significant proportion of health care in Canada took place in communities too small to support a specialist anesthesia practice. The eventual compromise was to define the practice of anesthesia as a "specialized field of medicine" while explicitly recognizing that "appropriately trained family physicians may be required to provide anesthesia services" in some communities. The role of anesthesia assistants (AA) was a similarly contentious issue. The role of paramedical personnel was first raised by Dr. Graves in a report to Council in 1968. The discussion of their role continued for many years and culminated in 2006 in a "Position Paper on Anesthesia Assistants" published by the Canadian Anesthesiologists' Society as Appendix 5 to the Guidelines. The Society remains involved in defining the curriculum for AA training in a joint working group 
with the Canadian Society of Respiratory Therapists and the Canadian Nurses Association.

In other areas, anesthesiologists saw the Guidelines as an instrument of "support". The gradual development of more extensive monitoring requirements has supported many anesthesia departments in their quests for funding from hospital administration for equipment purchases. Monitoring requirements have gradually progressed from the simple mention of recording "vital signs" to recommending oximetry, capnometry, and anesthetic agent monitoring.

Over the years, specialized areas of anesthetic practice, such as Obstetric Regional Anesthesia, Acute Pain Management, and Anesthesia Practice outside a Hospital, have received attention and specific reference. Consistent with the general philosophy, these specialized areas were not compiled as exhaustive level of evidence reviews but as practical and flexible guidelines that can be adapted to the practice of a local department.

In recent years, the Guidelines have been revised to recognize the importance of continuing education in all aspects of anesthesia, the value of information management services, and the significance of specific requirements for discharge of patients to home after day-care surgery. The revised Guidelines published in this issue of the Journal incorporate modifications to recognize changes in the organizational structure of health care, e.g., the term "hospital" is largely replaced with the term "health care facility". "Fast Track" recovery or postanesthesia care unit bypass has become common, and terminology has been amended to recognize that an alternative nursing care unit may be used for some aspects of immediate postoperative care. Finally, there are several editing changes where careful scrutiny has detected redundancies and duplication of terms.

By way of a membership survey and strategic planning analysis during 2008 and 2009, the Society undertook a detailed review of its core activities, its structure, and its deliverables to the membership. During this process, $90 \%$ of member respondents supported the Guidelines, and they were identified as one of the most important aspects of the Society's activities. The majority of respondents endorsed the Society's objective of providing broad principles of care through the Guidelines, and virtually all respondents reported having reviewed or used the published guidelines on at least a yearly basis. ${ }^{\mathrm{A}}$ The membership surveys included many suggestions for further improvements to the Guidelines, and these will be addressed by the Committee on Standards and the Society over the coming years. The new approach to publishing the Guidelines in the Journal is intended to facilitate improved archiving and easier access

A CAS Standards Committee. Report to CAS Board, June 2009. to both the printed and online versions of this document for all Society members and subscribers to the Journal.

\section{Annonce spéciale: Guide d'exercice de l'anesthésie-édition révisée 2010}

Le premier Guide des normes minimales d'exercice de l'anesthésie (le Guide) a été approuvé par le Conseil d'administration de la Société canadienne des anesthésiologistes le 17 janvier 1975 et publié sous forme de brochure en 1977. Plusieurs événements concomitants ont mené à la formulation de ce Guide. ${ }^{1}$ L'élaboration de normes en matière de «Services d'anesthésie » par le Conseil canadien d'agrément des hôpitaux (aujourd'hui Agrément Canada) a tout d'abord été reçu avec beaucoup d'appréhension dans le milieu de l'anesthésie; en effet, cela a été perçu comme un empiètement sur les «domaines de responsabilité ». Plus ou moins au même moment est survenu un décès malencontreux sous anesthésie, ce qui a poussé l'Association canadienne de protection médicale à consulter la Société et à lui conseiller de proposer des normes minimales d'exercice de l'anesthésie.

Il a incombé au comité des Normes de pratique de préparer ce guide et une ébauche finale, semblable au format que nous connaissons aujourd'hui, a été acceptée en 1974 et publiée en 1975 en tant que politique de la Société canadienne des anesthésiologistes. Le Docteur J. Earl Wynands, alors président de la Société, déclarait que le comité avait produit « un compte-rendu de premier plan et définitif qui aura un impact sur l'exercice et la prestation des soins en anesthésie au pays ». D'ailleurs, tout cela a eu lieu plusieurs années avant que les Normes de Harvard (Harvard Standards) soient proposées en $1986^{2}$; la Société peut donc être considérée comme un chef de file international dans les domaines de l'élaboration de normes et de la sécurité des patients.

Le Guide a été reçu avec enthousiasme, bien que le soutien dont il a alors été l'objet ne fût pas universel, certains exprimant leur inquiétude quand au fait que la Société avait « établi une norme qui devait désormais être respectée ». Toutefois, un consensus a vu le jour, admettant que l'objectif du Guide était de représenter la norme de soins généralement acceptée et qu'il servait de «stimulus à l'amélioration de la qualité de l'exercice de l'anesthésie ». Le Guide a été rédigé de façon à être suffisamment souple pour être modifié selon l'évolution de la pratique de l'anesthésie, des techniques de monitorage et de la mise en œuvre de nouvelles technologies. Le Guide a été mis à jour périodiquement, à intervalles irréguliers jusqu'en 1989 et 
annuellement depuis. Dans une des premières révisions du Guide, le terme de "norme » a été supprimé du titre du document, mettant plutôt l'emphase sur le terme « guide », afin d'indiquer que l'objectif du document était de représenter un "énoncé de principe plutôt qu'une norme ou qu'un niveau requis de conduite ».

Pendant quelque temps, le rôle des médecins généralistes dans la prestation de soins en anesthésie en plus de ceux fournis par des spécialistes formés par le Collège royal a constitué un point litigieux. Cette question est apparue au moment où l'on a pris conscience qu'une importante proportion des soins de santé au Canada étaient prodigués dans des communautés trop petites pour justifier une pratique de l'anesthésie spécialisée. Finalement, le compromis trouvé a été de définir la pratique de l'anesthésie comme un «domaine spécialisé de la médecine » tout en reconnaissant de façon explicite que «des médecins de famille formés adéquatement pourraient devoir fournir des services en anesthésie » dans certaines collectivités. Le rôle des assistants en anesthésie (AA) a également constitué un point de litige. Le rôle du personnel paramédical a été abordé pour la première fois par le Dr Graves dans un compte-rendu présenté au Conseil en 1968. La discussion quant à leur rôle a duré plusieurs années et a culminé en 2006 dans un «Exposé de principe sur les assistants en anesthésie », publié par la Société canadienne des anesthésiologistes (la Société) comme Annexe 5 du Guide. La Société est toujours impliquée dans l'élaboration du programme de formation des AA dans le cadre d'un groupe de travail conjoint avec la Société canadienne des thérapeutes respiratoires et l'Association des infirmières et infirmiers du Canada.

Dans d'autres domaines, les anesthésiologistes ont perçu le Guide comme un instrument de «soutien ». Le développement progressif de besoins plus poussés de monitorage a soutenu nombre de départements d'anesthésie dans leur recherche de financement provenant de l'administration de l'institution pour l'achat de matériel. Les besoins de monitorage ont petit à petit évolué de la simple mention d'enregistrer «les signes vitaux» à la recommandation d'un monitorage par oxymétrie, par capnométrie et du monitorage des agents anesthésiques.

Au fil des ans, des domaines spécialisés de la pratique de l'anesthésie, comme l'anesthésie régionale pour le travail obstétrical, la prise en charge de la douleur aiguë et la pratique de l'anesthésie hors hôpital, ont reçu une attention particulière et des mentions spécifiques. En ligne avec la philosophie générale, ces domaines spécialisés n'ont pas été compilés comme revues de données probantes exhaustives mais plutôt comme un guide pratique et souple pouvant s'adapter à la pratique d'un département local.
Ces dernières années, le Guide a été révisé de manière à rendre compte de l'importance de la formation continue dans tous les domaines de l'anesthésie, de la valeur des services de gestion de l'information, et de l'importance d'exigences spécifiques pour autoriser le congé des patients après une chirurgie ambulatoire. Le Guide révisé publié dans ce numéro du Journal intègre certaines modifications qui tiennent compte des changements de la structure organisationnelle des soins de santé; par exemple, le terme « hôpital » est la plupart du temps remplacé par le terme «établissement (de soins)». Le rétablissement « rapide » ou le transfert sans passage en salle de réveil est courant, et la terminologie a été modifiée en conséquence afin de reconnaître qu'une unité de soins infirmiers alternative peut être utilisée pour prodiguer des soins postopératoires immédiats dans certains cas. Enfin, plusieurs modifications éditoriales nous ont permis de supprimer les redondances et les duplications de termes.

En 2008 et 2009, la Société a réalisé un sondage auprès de ses membres ainsi qu'une analyse de planification stratégique. En regard des résultats de cet examen, elle a ensuite entrepris une révision détaillée de ses activités de base, de sa structure, et des publications et autres livrables qu'elle offrait à ses membres. Pendant le processus, $90 \%$ des répondants se sont exprimés en faveur du Guide, lequel a été identifié comme l'un des éléments les plus importants parmi les activités de la Société. La majorité des répondants approuve l'objectif de la Société de présenter des principes généraux de soins dans le Guide, et littéralement tous les répondants ont déclaré avoir révisé ou utilisé le guide publié au moins une fois par an. ${ }^{\mathrm{A}}$ Plusieurs suggestions d'améliorations du Guide sont revenues avec les sondages, et elles seront abordées par le Comité des normes et la Société dans les années à venir. L'objectif de notre nouvelle approche de publication du Guide, à savoir dans le Journal, est de faciliter un meilleur archivage et de rendre plus accessibles les versions imprimée et en ligne de ce document pour tous les membres de la Société et les abonnés du Journal.

Competing interests Dr. Richard Merchant is Chair of the Committee on Standards of the Canadian Anesthesiologists' Society.

\section{References}

1. Shephard DA. Watching closely those who sleep-a history of the Canadian Anaesthetists' Society 1943-1993. Can J Anaesth 1993; 40: 83-100.

2. Eichhorn JH, Cooper JB, Cullen DJ, Maier WR, Philip JH, Seeman $R G$. Standards for patient monitoring during anesthesia at Harvard Medical School. JAMA 1986; 256: 1017-20. 